\title{
A 3-manifold group which is not four dimensional linear
}

\author{
J. O. Button \\ Selwyn College \\ University of Cambridge \\ Cambridge CB3 9DQ \\ U.K. \\ j.o.button@dpmms.cam.ac.uk
}

\begin{abstract}
We give examples of closed orientable graph 3-manifolds having a fundamental group which is not a subgroup of $G L(4, \mathbb{F})$ for any field $\mathbb{F}$. This answers a question in the Kirby problem list from 1977 which is credited to the late William Thurston.
\end{abstract}

Keywords: 3-manifold, fundamental group, linear group 2000 MSC: 57M05, 20H20

\section{Introduction}

As part of Thurston's revolutionary understanding of 3 dimensional geometry and topology, he established that many 3-manifolds have hyperbolic structures. The fundamental group of a hyperbolic 3-manifold is a subgroup of $P S L(2, \mathbb{C})$ that lifts to $S L(2, \mathbb{C})$, so is linear (which here will always mean a subgroup of $G L(n, \mathbb{F})$ for $\mathbb{F}$ a field) in 2 dimensions. Now $S L(2, \mathbb{C}) \leq G L(4, \mathbb{R})$ because a $\mathbb{C}$-linear map of $\mathbb{C}^{2}$ is an $\mathbb{R}$-linear map of $\mathbb{R}^{4}$, so we can ask whether every finitely generated 3 -manifold group embeds in $G L(4, \mathbb{R})$. In fact this is Question 3.33 Part (A) in the first version of the Kirby problem list which dates from 1977 and is credited to Thurston, with Part (B) asking whether these groups are all residually finite. Now linearity of a finitely generated group implies residual finiteness and Part (B) was shown to be true on the acceptance of Perelman's solution to Geometrisation, because Thurston indicated and Hempel proved in [6] that residual finiteness is preserved when constructing 3-manifolds from their geometric pieces which themselves will have linear fundamental group. After this, Aschenbrenner 
and Friedl showed in [3] that finitely generated 3-manifold groups are virtually residually finite- $p$ for all but finitely many primes $p$. This can be seen as further evidence that all of these groups are linear because this property is implied by linearity but is stronger than being residually finite.

Now the question of linearity is still open for 3-manifold groups. However a large amount of recent activity using the work [12] of Wise, such as [8], [2] and [11], means it remains only to show linearity for those closed graph 3-manifolds which fail to possess a metric of non positive curvature, as then all compact prime 3-manifolds would have linear fundamental group and a free product of linear groups is linear.

However, even if all 3-manifold groups are linear, we can still ask the stronger question: is there $n \in \mathbb{N}$ such that all finitely generated 3-manifold groups embed in $G L(n, \mathbb{R})$ ? In this paper we show that we cannot take $n=4$, thus answering Thurston's Question 3.33 Part (A) in the negative. Indeed the answer is still negative even on replacing $\mathbb{R}$ with any field of any characteristic. We describe the 3-manifold in Section 2 and give details of its fundamental group but here we can summarise it thus: take two copies of the product of the punctured torus and the circle and form the graph manifold by identifying the boundary tori, with some conditions on the monodromy. The whole argument relies only on using Jordan normal form up to 4 by 4 matrices and considering the centraliser of a matrix in Jordan normal form. However the key idea is this: the circle in the first product 3-manifold requires a matrix having a big centraliser (by which we mean it contains a non abelian free group). But as we do not allow ourselves to identify the two circles, this centraliser cannot contain the whole 3-manifold group. This argument applies also to the circle on the other side and if these two elements are both diagonalisable then they are simultaneously diagonalisable as they commute. This forces a block structure for all the matrices in each of the two pieces of the graph manifold and in Section 3 we show by an easy examination of the possible cases for both block decompositions in 4 dimensions that this cannot occur, because the diagonal entries of the circle elements will be roots of unity so these elements will have finite order.

In Section 4 we show how this conclusion that the eigenvalues are roots of unity generalises to arbitrary matrices over an algebraically closed field, by replacing simultaneous diagonalisation by simultaneous triangularisation. This then allows the positive characteristic case to be eliminated first by a quick argument, leaving only the field $\mathbb{C}$ without loss of generality. However in Section 5 we now have to deal with the circle elements having more 
complicated Jordan normal forms. Although their eigenvalues are still roots of unity, such matrices may of course have infinite order in the characteristic zero case. This section requires some rather more specialised arguments which we feel would not extend quickly to dimensions above 4, unlike those in the earlier sections. However the basis of these arguments is just taking each possible Jordan normal form for the circle elements and working out the centralisers.

In the last section we make a few related comments, including noting that our graph manifolds have already appeared in the literature where they were shown not to have a metric of non positive curvature (so the linearity of these 3-manifold groups is still open) and to be non fibred but virtually fibred.

\section{Description of the graph manifolds}

We can form a closed orientable graph 3-manifold in the following way: let $S_{g, 1}$ be the compact orientable surface of genus $g \geq 1$ with one boundary component. We know that $\pi_{1}\left(S_{g, 1}\right)=F_{2 g}$, the free group of rank $2 g$, and we let $A \in F_{2 g}$ be the element given by the boundary curve (oriented in some way). On forming the product manifold $M_{1}=S_{g, 1} \times S^{1}$ (which can be regarded as a trivial Seifert fibre space) we have that the group $G_{1}=\pi_{1}\left(M_{1}\right)$ is isomorphic to $F_{2 g} \times \mathbb{Z}$ with the element $S$ generating $\mathbb{Z}$ being in the centre of this fundamental group. Moreover we have $\langle A, S\rangle=\mathbb{Z} \times \mathbb{Z}$ as this forms the fundamental group of the boundary torus $\partial M_{1}$.

We now take another manifold $M_{2}$ of this type with fundamental group $G_{2}$ (here the genus $g^{\prime}$ of our new surface $S_{g^{\prime}, 1}$ does not need to equal $g$, although in Section 5 we will require that $g=g^{\prime}=1$ ) with $B$ the corresponding peripheral element of $S_{g^{\prime}, 1}$ and $T$ the equivalent generator of the centre of $\pi_{1}\left(S_{g^{\prime}, 1} \times S_{1}\right)$. Let $M$ be the closed orientable graph manifold $M_{1} \#_{f} M_{2}$ where $f: \partial M_{1} \rightarrow \partial M_{2}$ is a homeomorphism of the torus which identifies the boundaries of the two 3-manifolds. This means that $\pi_{1}(M)$ is equal to the amalgamated free product $\left(F_{2 g} \times \mathbb{Z}\right) *_{\theta}\left(F_{2 g^{\prime}} \times \mathbb{Z}\right)$ where $\theta:\langle A, S\rangle \rightarrow\langle B, T\rangle$ is an isomorphism. The automorphisms of $\mathbb{Z} \times \mathbb{Z}$ are of course elements of $G L(2, \mathbb{Z})$ so we have integers $i, j, k, l$ with $i l-j k= \pm 1$ such that $B=A^{i} S^{j}$ and $T=A^{k} S^{l}$. Although it seems that the sign affects the orientability of $M$, we can assume that $i l-j k=1$ for $\pi_{1}(M)$ because we can replace $T$ by $T^{-1}$ (thus $k$ and $l$ by $-k$ and $-l$ ) without changing the group. For here on we do not consider 3-manifolds as we only need to examine the group $G=\pi_{1}(M)$, 
although we now must note the fact that $A$, and $B$, is equal to a product of commutators in $G_{1}$, respectively $G_{2}$.

\section{Diagonalisable peripheral elements over $\mathbb{C}$}

One of the most basic but useful facts in linear algebra is that if two $n \times n$ matrices $X, Y$ with entries in a field $\mathbb{F}$ are separately diagonalisable over $\mathbb{F}$ and they commute then they are simultaneously diagonalisable. This is because $Y$ maps the $\lambda$-eigenspace $E_{\lambda}(X)=\left\{v \in \mathbb{F}^{n}: X v=\lambda v\right\}$ of $X$ into itself and so we can diagonalise $Y$ when restricted to each $E_{\lambda}(X)$. Thus on taking our group $G$, we assume for the remainder of this section that both the peripheral elements $A$ and $S$ of $\pi_{1}\left(M_{1}\right)$ are diagonalisable over $\mathbb{F}$, with the other cases being dealt with in the later sections. We take $\mathbb{F}=\mathbb{C}$ for definiteness here and as the case of most interest, but the arguments are valid in any field on interpreting the phrase 'root of unity' as an element of finite order in the multiplicative group $\mathbb{F}-\{0\}$. Later in the paper we will require that none of $i, j, k, l \in \mathbb{Z}$ are equal to zero.

Consequently if $G$ embeds as a subgroup of $G L(n, \mathbb{C})$ for some $n$, we can conjugate $G$ so that both $A$ and $S$ are diagonal matrices, as well as $B$ and $T$ which are products of these two elements. Now $S$ (and also $T$ ) must have a repeated eigenvalue, because a diagonal element in $G L(n, \mathbb{C})$ with all entries distinct can only commute with other diagonal elements, thus its centraliser is abelian whereas the centraliser of $S$ contains the group $G_{1}$.

Proposition 3.1. Let $\lambda_{1}, \ldots, \lambda_{c}$ be the distinct eigenvalues of $S$ (hence $c<$ $n)$ with eigenspaces $U_{\lambda_{1}}, \ldots, U_{\lambda_{c}}$ of dimensions $\gamma_{1}, \ldots, \gamma_{c}$ respectively and let $\mu_{1}, \ldots, \mu_{d}$ and $V_{\mu_{1}}, \ldots, V_{\mu_{d}}$ of dimensions $\delta_{1}, \ldots, \delta_{d}$ be the equivalent for $T$ (with $d<n$ also).

If $e_{1}, \ldots, e_{n}$ is any basis which simultaneously diagonalises $S$ and $A$ (hence also $T$ and $B)$ then there is a permutation $\sigma$ of $\{1, \ldots, n\}$ such that

$\left\langle e_{\sigma(1)}, \ldots, e_{\sigma\left(\gamma_{1}\right)}\right\rangle=U_{\lambda_{1}},\left\langle e_{\sigma\left(\gamma_{1}+1\right)}, \ldots, e_{\sigma\left(\gamma_{1}+\gamma_{2}\right)}\right\rangle=U_{\lambda_{2}}, \ldots,\left\langle e_{\sigma\left(n-\gamma_{c}+1\right)}, \ldots, e_{\sigma(n)}\right\rangle=U_{\lambda_{c}}$

and also a permutation $\tau$ of $\{1, \ldots, n\}$ such that

$\left\langle e_{\tau(1)}, \ldots, e_{\tau\left(\delta_{1}\right)}\right\rangle=V_{\mu_{1}},\left\langle e_{\tau\left(\delta_{1}+1\right)}, \ldots, e_{\tau\left(\delta_{1}+\delta_{2}\right)}\right\rangle=U_{\mu_{2}}, \ldots,\left\langle e_{\tau\left(n-\delta_{d}+1\right)}, \ldots, e_{\tau(n)}\right\rangle=U_{\mu_{d}}$.

Furthermore the subspaces $U_{\lambda_{1}}, \ldots, U_{\lambda_{c}}$ are invariant under any element of $G_{1}$, and similarly for $V_{\mu_{1}}, \ldots, V_{\mu_{d}}$ with $G_{2}$. 
Proof. As both $S$ and $T$ are diagonalisable we have

$$
\mathbb{C}^{n}=\left\langle e_{1}\right\rangle \oplus \cdots \oplus\left\langle e_{n}\right\rangle=U_{\lambda_{1}} \oplus \cdots \oplus U_{\lambda_{c}}=V_{\mu_{1}} \oplus \cdots \oplus V_{\mu_{d}} .
$$

But as they are simultaneously diagonalisable with respect to $e_{1}, \ldots, e_{n}$ we have that each $e_{i}$ is both an eigenvector of $S$ and of $T$, so $e_{i}$ belongs in one of $U_{\lambda_{1}}, \ldots, U_{\lambda_{c}}$ and in one of $V_{\mu_{1}}, \ldots, V_{\mu_{d}}$. However we cannot have more than $\gamma_{j}$ elements of the basis $e_{1}, \ldots, e_{n}$ in $U_{\lambda_{j}}$ (and more than $\delta_{j}$ in $V_{\mu_{j}}$ ) by linear independence, so each $U_{\lambda_{i}}$ is a direct sum of a subset of $e_{1}, \ldots, e_{n}$ whereupon we obtain our permutation $\sigma$ (and separately the permutation $\tau$ ).

Finally on being given any $g \in G_{1}$ and $u \in U_{\lambda_{i}}$ we have $g S=S g$ so $g S(u)=\lambda_{i} g(u)=S(g(u))$, placing $g(u)$ in $U_{\lambda_{i}}$.

The idea now is to look at the equations satisfied by the diagonal entries of the four matrices $A, B, S, T$. We have $2 n+c+d$ variables in these entries, coming from each of the $n$ diagonal entries of $A$ and $B$, along with the $c$ eigenvalues for $S$ and $d$ for $T$. We also have the identities $B=A^{i} S^{j}$, $T=A^{k} S^{l}$ providing $2 n$ equations, which are linear homogeneous equations for elements in the abelian group $\mathbb{C}-\{0\}$, written multiplicatively. Therefore it seems we ought to be able to find non trivial solutions to these equations, but we also need to recall that $A$ and $B$ are elements in the commutator subgroup of $G_{1}$ and $G_{2}$ respectively. As the determinant is a homomorphism from $G L(n, \mathbb{C})$ to $\mathbb{C}-\{0\}$, we must have $\operatorname{det}(A)=\operatorname{det}(B)=1$. Moreover as all elements of $G_{1}$ have the same block structure (at least on applying the permutation $\sigma$ ) by the last part of Proposition 3.1, each block of $A$ is also a product of commutators in $G L(m, \mathbb{C})$ for the relevant $m<n$ and so they all have determinant 1 as well. The same applies to $B$ so we now have $c+d$ further homogeneous equations to satisfy, thus we no longer can guarantee the existence of non trivial solutions.

We have seen that $c, d<n$ and we also note that $c$ (and $d$ ) is greater than 1 as otherwise $S$ (or $T$ ) would be a scalar multiple $\lambda I_{n}$ of the identity. But $B=A^{i} S^{j}$ with $\operatorname{det}(A)$ and $\operatorname{det}(B)$ equal to 1 , which implies (if $j \neq 0$ ) that $\operatorname{det}(S)$ is a root of unity. Thus $\lambda$ is a root of unity too which means that $S$ has finite order. Similarly the decomposition of $\mathbb{C}^{n}$ into the $T$-eigenspaces $\left\{V_{\mu_{t}}: 1 \leq t \leq d\right\}$ cannot be the same as that for the $S$-eigenspaces $\left\{U_{\lambda_{s}}\right.$ : $1 \leq s \leq c\}$, nor can we have every $V_{\mu_{t}}$ contained in some $U_{\lambda_{s}}$ for $s$ depending on $t$. Otherwise every element of $G_{1}$ and $G_{2}$ preserves the $S$-eigenspaces, 
thus the restrictions of both $A$ and $B$ to each $U_{\lambda_{s}}$ have determinant 1 and the argument just given applies to show that the eigenspace $U_{\lambda_{s}}$ corresponds to an eigenvalue which is a root of unity. As this applies for all $U_{\lambda_{s}}$, once again $S$ has finite order. Of course the same holds with $S$ and $T$ swapped.

As a warm up we first consider the lower dimensions. For two dimensions, note that $G_{1}=F_{2 g} \times \mathbb{Z}$ does embed in $G L(2, \mathbb{C})$ but only by taking $S$ equal to $\lambda I$ (for $\lambda$ not a root of unity) so this would imply that $S$ commutes with all of $G$.

In higher dimensions, we actually prove here that if there is an embedding of $G$ into $G L(3, \mathbb{C})$ in which $A$ and $S$ are diagonalisable then all eigenvalues of the peripheral elements $A, B, S, T$ must be roots of unity. This implies that these peripheral elements would have finite order which yields a contradiction. We state our result in this form because we will also need to establish this fact about eigenvalues of the peripheral elements in the later sections when we consider the non diagonalisable case.

Proposition 3.2. Suppose that the group $G$ as above with $j \neq 0$ embeds in $G L(3, \mathbb{C})$ such that the elements $A$ and $S$ are both diagonalisable over $\mathbb{C}$. Then all eigenvalues of the elements $A, B, S, T$ are roots of unity, thus such an embedding cannot exist.

Proof. If $A$ and $S$ are both diagonalisable then we can choose a basis $e_{1}, e_{2}, e_{3}$ such that they are simultaneously diagonalisable. Now $S$ must have one double and one single eigenvalue $\lambda_{1}, \lambda_{1}, \lambda_{2}$ say (else it commutes with either everything or only diagonal elements) and the same is true for $T$ with eigenvalues $\mu_{1}, \mu_{1}, \mu_{2}$ say. Thus by Proposition 3.1 we can permute $e_{1}, e_{2}, e_{3}$ such that we have $U_{\lambda_{1}}=\left\langle e_{1}, e_{2}\right\rangle$ and $U_{\lambda_{2}}=\left\langle e_{3}\right\rangle$ for the two $S$-eigenspaces, meaning that all elements of $G_{1}$ have block structure $\left\langle e_{1}, e_{2}\right\rangle \oplus\left\langle e_{3}\right\rangle$.

Also by this Proposition we know that two of $e_{1}, e_{2}, e_{3}$ span $V_{\mu_{1}}$ and the other spans $V_{\mu_{2}}$. But we cannot have the same block structures for $S$ and $T$ by our comment above, so we cannot have $e_{3} \in V_{\mu_{2}}$. Although we are not now free to permute the basis elements arbitrarily, we can swap $e_{1}$ and $e_{2}$ if necessary and can assume that $V_{\mu_{2}}=\left\langle e_{2}\right\rangle$, so we have $\left\langle e_{1}, e_{3}\right\rangle \oplus\left\langle e_{2}\right\rangle$ for the block structure of all elements of $G_{2}$.

On imposing the determinant condition for each block of $A$ and $B$, our four peripheral elements must have the following form, where we are writing 
diagonal matrices as column vectors:

$$
A=\left(\begin{array}{r}
a \\
1 / a \\
1
\end{array}\right), S=\left(\begin{array}{c}
\lambda \\
\lambda \\
\mu
\end{array}\right) ; B=\left(\begin{array}{r}
\alpha \\
1 \\
1 / \alpha
\end{array}\right), T=\left(\begin{array}{c}
\eta \\
\theta \\
\eta
\end{array}\right)
$$

for $a, \lambda, \mu, \alpha, \eta, \theta \in \mathbb{C}-\{0\}$. Now the identity $B=A^{i} S^{j}$ implies that $(\operatorname{det} S)^{j}=1$. For the moment we assume that all complex roots of unity are equal to one and thus we take $\mu=1 / \lambda^{2}$. Then the double appearance of $\eta$ in $T$ implies that $a^{k} \lambda^{3 l}=1$ but the 1 in $B$ means that $a^{i}=\lambda^{j}$, thus $\lambda^{k j+3 l i}=1$. As $k j+3 l i=4 l i-1$, this means that $\lambda$ (and from here all other variables) is a root of unity unless $l i=1 / 4$ which cannot happen.

To deal with roots of unity in general, suppose that we have found a solution to the entries of $A, S, B, T$ in which roots of unity appear. Let $N$ be the least common multiple over all the orders of these roots. Then on replacing $A, S, B, T$ with $A^{N}, S^{N}, B^{N}, T^{N}$ the same equations between these matrices continue to hold, as well as the determinant being 1 in each block of $A^{N}$ and $B^{N}$, but now all roots of unity have been replaced by 1 .

Theorem 3.3. Suppose that the group $G$ above embeds in $G L(4, \mathbb{C})$ with $A$ and $S$ both diagonalisable and none of $i, j, k, l$ equal to 0 . Then all eigenvalues of the elements $A, B, S, T$ are roots of unity, thus such an embedding does not exist.

Proof. After the simultaneous diagonalisation we have both the block structure for $G_{1}$ and for $G_{2}$. The possibilities for the block sizes on either side, which need not be the same, are $(3,1),(2,2)$ and $(2,1,1)$. We first note that if the same block of size 1 appears in both $G_{1}$ and $G_{2}$ then there is an entry of 1 in the same place of $A$ and of $B$ (coming from the determinant condition), thus $A^{i} S^{j}=B$ means that the equivalent entry of $S$ is also (a root of) unity, and thus so is that of $T$ using $A^{k} S^{l}=T$. Thus on taking this block of size 1 and the complementary block of size $n-1$ (which here is 3 ), we obtain an expression of $G$ as a subdirect product inside $H_{1} \times H_{2}$, where $H_{1}$ is the image of $G$ under the homomorphism which is restriction to the size 1 block, and similarly $H_{2}$ for the size $n-1$ block. But $H_{1}$ being abelian implies that the latter homomorphism is an isomorphism, because an element of $G$ that restricts to $I_{n-1}$ will be in the centre of $G$. Consequently we we can delete 
the size 1 block from our matrices without changing the group, thus reducing the problem to one lower dimension which is covered by Proposition 3.2.

We now outline the argument in each of the possible cases and will henceforth write all equations additively.

Case 1: Both blocks are $(2,2)$.

Let the decomposition for $S$ be $\left\langle e_{1}, e_{2}\right\rangle \oplus\left\langle e_{3}, e_{4}\right\rangle$. As noted earlier, this cannot be the same for $T$ so without loss of generality we set this to be $\left\langle e_{1}, e_{4}\right\rangle \oplus\left\langle e_{2}, e_{3}\right\rangle$. This gives rise to diagonal matrices

$$
A=\left(\begin{array}{r}
a \\
-a \\
b \\
-b
\end{array}\right), S=\left(\begin{array}{c}
\lambda \\
\lambda \\
\mu \\
\mu
\end{array}\right) ; B=\left(\begin{array}{r}
\alpha \\
\beta \\
-\beta \\
-\alpha
\end{array}\right), T=\left(\begin{array}{c}
\eta \\
\theta \\
\theta \\
\eta
\end{array}\right) .
$$

As before, considering determinants of $S$ and $T$, assuming $j \neq 0$, and ignoring roots of unity gives $\mu=-\lambda$ and $\theta=-\eta$. Then the appearance of $\pm \alpha$ at the top and bottom of $B$ implies $i a+j \lambda=\alpha=i b+j \lambda$, thus $a=b$ as $i \neq 0$. But now the two $\eta$ s in $T$ give $k a+l \lambda=\eta=-k a-l \lambda$ so $\eta=0=\theta$, meaning that $T$ is the identity (or a power of $T$ is on removing roots of unity). Now the top two equations for the entries of $T$ tell us that $a$ and $\lambda$ are also roots of unity, so everything is.

Case 2: Both blocks are $(3,1)$.

The comment at the start of this proof shows that we do not need to consider the case where the two decompositions are the same. Consequently we can without loss of generality set them equal to $\left\langle e_{1}, e_{2}, e_{3}\right\rangle \oplus\left\langle e_{4}\right\rangle$ for $S$ and $\left\langle e_{1}, e_{2}, e_{4}\right\rangle \oplus\left\langle e_{3}\right\rangle$ for $T$, giving entries

$$
A=\left(\begin{array}{r}
a \\
b \\
-a-b \\
0
\end{array}\right), S=\left(\begin{array}{c}
\lambda \\
\lambda \\
\lambda \\
-3 \lambda
\end{array}\right) ; B=\left(\begin{array}{r}
\alpha \\
\beta \\
0 \\
-\alpha-\beta
\end{array}\right), T=\left(\begin{array}{c}
\eta \\
\eta \\
-3 \eta \\
\eta
\end{array}\right)
$$

where we have set all determinants equal to 1 , as $j \neq 0$. Also $k \neq 0$ gives $a=b$ by examining the top two entries of $T$ so $\alpha=\beta$. Thus $k a+l \lambda=\eta=-3 l \lambda$ and $-2 i a+j \lambda=0$, giving $(8 i l+j k) \lambda=0$. As $9 i l \neq 1$ we have $S=I$.

Case 3: Both blocks are $(2,1,1)$.

As we do not need to put blocks of size 1 together, we will take $\left\langle e_{1}, e_{4}\right\rangle \oplus$ 
$\left\langle e_{2}\right\rangle \oplus\left\langle e_{3}\right\rangle$ for $S$ and $\left\langle e_{2}, e_{3}\right\rangle \oplus\left\langle e_{1}\right\rangle \oplus\left\langle e_{4}\right\rangle$ for $T$ and set

$$
A=\left(\begin{array}{r}
a \\
0 \\
0 \\
-a
\end{array}\right), S=\left(\begin{array}{c}
\lambda \\
\mu \\
\nu \\
\lambda
\end{array}\right) ; B=\left(\begin{array}{r}
0 \\
\alpha \\
-\alpha \\
0
\end{array}\right), T=\left(\begin{array}{l}
\theta \\
\eta \\
\eta \\
\nu
\end{array}\right)
$$

which gives $i a+j \lambda=0=-i a+j \lambda$, forcing $A=I$ and all entries are again roots of unity.

Case 4: Blocks of form $(2,2)$ and $(2,1,1)$.

We can swap $G_{1}$ and $G_{2}$ if necessary so that $S$ has the $(2,2)$ blocks. This has the effect of replacing the matrix $\left(\begin{array}{cc}i & j \\ k & l\end{array}\right)$ with its inverse, so the entries will still be non zero. Then we have without loss of generality

$$
A=\left(\begin{array}{r}
a \\
-a \\
b \\
-b
\end{array}\right), S=\left(\begin{array}{c}
\lambda \\
\lambda \\
-\lambda \\
-\lambda
\end{array}\right) ; B=\left(\begin{array}{r}
\alpha \\
0 \\
0 \\
-\alpha
\end{array}\right), T=\left(\begin{array}{c}
\theta \\
\eta \\
\nu \\
\theta
\end{array}\right)
$$

so the middle two entries of $B$ give $a=b$ and then the outer entries of $T$ imply $\theta=0$. But now we have $i a=j \lambda$ and $k a+l \lambda=0$ which means that $(2 i l-1) a=0$ so again $A=I$ and we only have roots of unity.

Case 5: Blocks of form $(3,1)$ and $(2,1,1)$.

Here we can assume we have blocks $\left\langle e_{1}, e_{2}, e_{3}\right\rangle \oplus\left\langle e_{4}\right\rangle$ for $S$ and $\left\langle e_{1}, e_{4}\right\rangle \oplus$ $\left\langle e_{2}\right\rangle \oplus\left\langle e_{3}\right\rangle$ for $T$ and set

$$
A=\left(\begin{array}{r}
a \\
b \\
-a-b \\
0
\end{array}\right), S=\left(\begin{array}{c}
\lambda \\
\lambda \\
\lambda \\
-3 \lambda
\end{array}\right) ; B=\left(\begin{array}{r}
\alpha \\
0 \\
0 \\
-\alpha
\end{array}\right), T=\left(\begin{array}{c}
\theta \\
\eta \\
\nu \\
\theta
\end{array}\right)
$$

which implies using $\alpha$ that $i a=2 j \lambda$ and $k a+4 l \lambda=0$ using $\theta$, so $(2 k j+4 i l) \lambda=$ 0 but $6 i l \neq 2$ so $S=I$ and only roots of unity appear.

Case 6: Blocks of form $(3,1)$ and $(2,2)$.

On setting blocks of $\left\langle e_{1}, e_{2}, e_{3}\right\rangle \oplus\left\langle e_{4}\right\rangle$ and $\left\langle e_{1}, e_{2}\right\rangle \oplus\left\langle e_{3}, e_{4}\right\rangle$ we obtain

$$
A=\left(\begin{array}{r}
a \\
b \\
-a-b \\
0
\end{array}\right), S=\left(\begin{array}{c}
\lambda \\
\lambda \\
\lambda \\
-3 \lambda
\end{array}\right) ; B=\left(\begin{array}{r}
\alpha \\
-\alpha \\
\beta \\
-\beta
\end{array}\right), T=\left(\begin{array}{c}
\theta \\
\theta \\
\eta \\
\eta
\end{array}\right)
$$


so the repeated $\theta$ gives $a=b$ and the repeated $\beta$ gives $2 i a+2 j \lambda=0$, but the repeated $\eta$ implies $2 a k=4 l \lambda$ so $(6 i l-2) \lambda=0$, giving $S=I$ with all roots of unity again.

Finally in this section we note that although we have written out the proof for $\mathbb{C}$, we can deal with all other fields as well.

Corollary 3.4. Suppose that $\mathbb{F}$ is any field and $\overline{\mathbb{F}}$ is its algebraic closure. If $G$ embeds in $G L(4, \overline{\mathbb{F}})$ with $i, j, k, l \neq 0$ and with $A$ and $S$ both diagonalisable over $\overline{\mathbb{F}}$ then all eigenvalues of the elements $A, B, S, T$ are roots of unity, thus such an embedding does not exist.

Proof. In Theorem 3.3 we were effectively showing that $U x=0$ implies $x=0$ where $x \in \mathbb{C}^{m}$ for the appropriate value of $m$ and $U$ was equal to various $m$ by $m$ matrices with entries in $\mathbb{Z}$ given by the gluing and determinant equations. Therefore we have shown that $\operatorname{det}(U) \neq 0$ and thus there is $V=\operatorname{adj}(U)$ such that $V U=\lambda I_{m}$ for $\lambda \in \mathbb{Z}-\{0\}$. Now suppose that $x$ is a vector with entries in an abelian group, thus in a module over $\mathbb{Z}$. If this $x$ satisfies $U x=0$ then $V U x=0=\lambda x$, thus every entry of $x$ has finite order dividing $\lambda$. On setting our abelian group to be the multiplicative group $\overline{\mathbb{F}}^{*}$, all eigenvalues of $A, B, S, T$ are just linear combinations of the entries in $x$, thus also have order dividing $\lambda$ and so are roots of unity in $\overline{\mathbb{F}}$.

\section{Diagonal entries in the non diagonal case}

We assumed throughout the last section that our peripheral elements could be diagonalised but now we will see that in the general case we can still use standard linear algebra to conclude that all eigenvalues of these peripheral elements are again roots of unity.

Given any element $X \in G L(n, \mathbb{C})$, or if given an arbitrary field $\mathbb{F}$ we can replace $\mathbb{C}$ by the relevant algebraic closure $\overline{\mathbb{F}}$, we know as part of the theory of Jordan normal form that $\mathbb{C}^{n}$ is spanned by its generalised eigenspaces

$$
G_{\lambda}(X)=\left\{v \in \mathbb{C}^{n}:(X-\lambda I)^{m} v=0 \text { for some } m \in \mathbb{N}\right\} .
$$


Now on taking another $Y \in G L(n, \mathbb{C})$ such that $X Y=Y X$, we have $Y\left(G_{\lambda}(X)\right) \subseteq G_{\lambda}(X)$ because $Y$ also commutes with $(X-\lambda I)^{m}$. Thus if $V_{1} \oplus \cdots \oplus V_{e}$ is the decomposition of the peripheral element $S$ into its generalised eigenspaces then this also provides a block decomposition for all elements in the group $G_{1}$. Thus not only does this apply to $A \in G_{1}$ but it also means that each square block in $A$ has determinant 1 as before. Unlike the diagonalisable case though, it is not guaranteed that an element which decomposes into these blocks commutes with $S$.

Now we consider $T=A^{k} S^{l}$ which also commutes with $S$, thus preserves the decomposition $V_{1} \oplus \cdots \oplus V_{e}$. Hence on restricting $S$ and $T$ to each $V_{c}$, this subspace further splits into a decomposition $V_{c}=W_{c, 1} \oplus \cdots \oplus W_{c, n_{c}}$ where $W_{c, m}$ is contained in a single generalised eigenspace of $T$. As $W_{c, m}$ is the intersection of a generalised eigenspace for $S$ and that for $T$, it is invariant under $S, T$ and any element commuting with both of these. We also note that as any element $g \in G_{2}$ commutes with $T$, the decomposition $V_{1}^{\prime} \oplus \cdots \oplus V_{e^{\prime}}^{\prime}$ into generalised eigenspaces for $T$ also provides a sum into square blocks for $g$. Thus as before each block of $B$ is a commutator and so has determinant 1.

We now restrict $A$ and $S$ to each subspace $W_{c, m}$ and this is invariant under both maps. Although our matrices need not be diagonalisable, we know that over $\mathbb{C}$ (or $\overline{\mathbb{F}}$ ) any matrix can be conjugated to be upper triangular. We would like to have an equivalent version of the "commute and diagonalisable implies simultaneously diagonalisable" result in the upper triangular case and this can be done (going under the name "simultaneous triangularisability" which is sometimes credited to Frobenius). One can indeed replace "diagonalisable" with "upper triangularisable" and "simultaneously diagonalisable" with "simultaneously upper triangularisable" in the above for two commuting matrices $X, Y$ because we have a non trivial eigenspace $E_{\lambda}(X)$ containing the first element of a basis making $X$ upper triangular. This eigenspace is invariant under $Y$, so on restricting $Y$ we obtain a common eigenvector for $X$ and $Y$. By taking this as our first basis vector, we can now take a quotient space and reduce the dimension by one, then continue by induction.

We now apply this result to $A$ and $S$ restricted to each $W_{c, m}$ and put these bases together to obtain a basis for $\mathbb{C}^{n}$ where these two matrices are upper triangular. This means that $B=A^{i} S^{j}$ and $T=A^{k} S^{l}$ are also upper triangular with the equations for the diagonal elements of $B$ and $T$ involving only the diagonal elements of $A$ and $S$, so these equations will be exactly 
the same as in the last section. Moreover the determinant equations for $A$ and $B$ will involve restricting to a basis for each $V_{c}$, respectively $V_{c}^{\prime}$, and we can do this by taking the appropriate elements of the basis from each $W_{c, m}$. Thus we are taking determinants of upper triangular subblocks which is just the product of diagonal terms, so any set of variables and equations for the diagonal elements has already appeared in Section 3. This gives us:

Theorem 4.1. If our group $G$ embeds in $G L(n, \mathbb{F})$ for any field $\mathbb{F}$ with algebraic closure $\overline{\mathbb{F}}$ then it can be conjugated in $G L(n, \overline{\mathbb{F}})$ so that the peripheral elements $A, S$ (and hence $B, T$ ) are upper triangular with all diagonal elements roots of unity in $\overline{\mathbb{F}}$.

Proof. As just mentioned, we can assume by conjugating $G$ that $A$ and $S$ are both upper triangular. The argument given before Proposition 3.2 that the number of eigenspaces of $S$ (and $T$ ) is strictly between 1 and $n$ still applies here, as does the argument that not every eigenspace of $T$ is contained in an $S$-eigenspace (or vice versa) because any determinant which needs to be evaluated will just be a product of elements on the diagonal of $A, S, B$ or $T$. Thus the proof of Theorem 3.3 showing that the only solutions to the equations for the diagonal elements are roots of unity applies here too.

We are now in a position to eliminate $G$ being a subgroup of $G L(4, \mathbb{F})$ when $\mathbb{F}$ has positive characteristic. It is well known in this case that if $X$ is a matrix in $G L(n, \mathbb{F})$ with all eigenvalues roots of unity in $\overline{\mathbb{F}}$ then $X$ has finite order, because we can conjugate in $G L(n, \overline{\mathbb{F}})$ so that a power of $X$ is upper uni-triangular and then use the binomial theorem. This gives us:

Corollary 4.2. Our group $G$ does not embed in $G L(4, \mathbb{F})$ if $\mathbb{F}$ is any field of positive characteristic.

Proof. On applying Theorem 4.1, we see that $A, S, B, T$ would have finite order.

\section{The characteristic zero case}

If $G$ embeds in $G L(4, \mathbb{F})$ for $\mathbb{F}$ a field of characteristic zero then we can say without loss of generality that $\mathbb{F}=\mathbb{C}$. This is because $G$ is finitely generated 
so we can embed $\mathbb{Q}\left(x_{1}, \ldots, x_{n}\right)$ into $\mathbb{C}$, where $x_{1}, \ldots, x_{n}$ are the matrix entries of a generating set for $G$. Unfortunately we are not yet finished because of matrices such as $\left(\begin{array}{ll}1 & 1 \\ 0 & 1\end{array}\right)$ which have infinite order despite all eigenvalues being roots of unity. We will need to develop some rather ad hoc arguments in this section in order to finish our proof (as well as introducing parity constraints on $i, j, k, l$ to avoid having to treat too many cases). Moreover at this point we set the genera $g, g^{\prime}$ of the two surfaces in Section 2 equal to 1 so that the peripheral elements $A$ and $B$ are now commutators and not just products of commutators. This now means we can apply the following facts about the commutator of a pair of 2 by 2 matrices, though we relegate proofs to the appendix.

Proposition 5.1. Given $\alpha, \beta \in G L(2, \mathbb{C})$, let $\gamma \in S L(2, \mathbb{C})$ be the commutator $\alpha \beta \alpha^{-1} \beta^{-1}$ and suppose that $\gamma$ has repeated eigenvalues which must therefore be both 1 or both -1, thus on conjugating we can assume that $\gamma= \pm\left(\begin{array}{ll}1 & b \\ 0 & 1\end{array}\right)$ for $b \in \mathbb{C}$

(i) If $\gamma=\left(\begin{array}{ll}1 & b \\ 0 & 1\end{array}\right)$ for $b \neq 0$ then both $\alpha$ and $\beta$ are already upper triangular too, thus $\langle\alpha, \beta\rangle$ is a soluble group.

(ii) If $\alpha, \beta$ are in $S L(2, \mathbb{C})$ and of infinite order with $\gamma=\left(\begin{array}{rr}-1 & -b \\ 0 & -1\end{array}\right)$ for some $b \neq 0$ then we can simultaneously conjugate $\alpha$ and $\beta$ in $S L(2, \mathbb{C})$ such that $\alpha \beta \alpha^{-1} \beta^{-1}$ is as above (though for a possibly different $b \in \mathbb{C}$ ) and

$$
\alpha^{-1} \beta^{-1} \alpha \beta=\left(\begin{array}{rr}
-1 & 0 \\
b & -1
\end{array}\right)
$$

whereupon there exist $z, w \in \mathbb{C}-\{0\}$ such that

$$
\alpha=\left(\begin{array}{cc}
\frac{1+z^{2}}{w} & z \\
z & w
\end{array}\right), \quad \beta=\left(\begin{array}{cc}
\frac{1+w^{2}}{z} & -w \\
-w & z
\end{array}\right) \text { with } b=\frac{2\left(1+z^{2}+w^{2}\right)}{z w} .
$$

We note here the following obvious but useful fact:

Lemma 5.2. If we have a homomorphism from a non abelian free group $F$ to $G L(n, \mathbb{C})$ with soluble kernel then this homomorphism is actually injective.

Proof. Any subgroup of $F$ is free so the kernel must be cyclic. However a standard fact about free groups is that the only normal finitely generated 
subgroup of infinite index is the identity.

We first sort the possible Jordan blocks (generalised eigenspaces with a suitable basis putting them into a canonical form) that can appear in a 4 by 4 matrix into 2 categories according to their centraliser.

Proposition 5.3. Given a Jordan block of size at most 4 by 4 , the following have a small centraliser (meaning does not contain a non abelian free group):

$(\lambda),\left(\begin{array}{cc}\lambda & 1 \\ 0 & \lambda\end{array}\right),\left(\begin{array}{ccc}\lambda & 1 & 0 \\ 0 & \lambda & 0 \\ 0 & 0 & \lambda\end{array}\right),\left(\begin{array}{ccc}\lambda & 1 & 0 \\ 0 & \lambda & 1 \\ 0 & 0 & \lambda\end{array}\right),\left(\begin{array}{cccc}\lambda & 1 & 0 & 0 \\ 0 & \lambda & 1 & 0 \\ 0 & 0 & \lambda & 0 \\ 0 & 0 & 0 & \lambda\end{array}\right),\left(\begin{array}{cccc}\lambda & 1 & 0 & 0 \\ 0 & \lambda & 1 & 0 \\ 0 & 0 & \lambda & 1 \\ 0 & 0 & 0 & \lambda\end{array}\right)$,

and these have big centraliser (they contain a non abelian free group):

$$
\lambda I_{2}, \lambda I_{3}, \lambda I_{4},\left(\begin{array}{cccc}
\lambda & 1 & 0 & 0 \\
0 & \lambda & 0 & 0 \\
0 & 0 & \lambda & 0 \\
0 & 0 & 0 & \lambda
\end{array}\right),\left(\begin{array}{cccc}
\lambda & 1 & 0 & 0 \\
0 & \lambda & 0 & 0 \\
0 & 0 & \lambda & 1 \\
0 & 0 & 0 & \lambda
\end{array}\right)
$$

Proof. Given one of these block matrices $M$, we can pre- and post-multiply $M-I$ (which has the same centraliser as $M$ ) by a general matrix and equate the two products. In particular any matrix with all 1s on the upper diagonal can only commute with particular upper triangular matrices and these will generate a soluble subgroup.

However in the other (non diagonal) cases we will change the canonical form to obtain a neater description of the centraliser which is easily checked. We replace

$$
\left(\begin{array}{lll}
0 & 1 & 0 \\
0 & 0 & 0 \\
0 & 0 & 0
\end{array}\right) \text { and }\left(\begin{array}{cccc}
0 & 1 & 0 & 0 \\
0 & 0 & 1 & 0 \\
0 & 0 & 0 & 0 \\
0 & 0 & 0 & 0
\end{array}\right)
$$

with the respective conjugate forms

$$
\left(\begin{array}{lll}
0 & 0 & 1 \\
0 & 0 & 0 \\
0 & 0 & 0
\end{array}\right) \text { and }\left(\begin{array}{cccc}
0 & 0 & 1 & 0 \\
0 & 0 & 0 & 0 \\
0 & 0 & 0 & 1 \\
0 & 0 & 0 & 0
\end{array}\right)
$$


which are both now easily confirmed by direct calculation to have a small centraliser, consisting only of upper triangular matrices.

For the matrices with big centraliser, we also also need to know the exact description of each centraliser. Whilst this is obvious for the scalar matrices, we will conjugate the penultimate matrix into the form

$$
\left(\begin{array}{cccc}
\lambda & 0 & 0 & 1 \\
0 & \lambda & 0 & 0 \\
0 & 0 & \lambda & 0 \\
0 & 0 & 0 & \lambda
\end{array}\right) \text {, which has centraliser }\left(\begin{array}{cccc}
a & ? & ? & ? \\
0 & ? & ? & ? \\
0 & ? & ? & ? \\
0 & 0 & 0 & a
\end{array}\right)
$$

where ? denotes any complex number, not necessarily the same number on each appearance, whereas repeated letters are equal to each other. Meanwhile the final matrix will instead be written

$$
\left(\begin{array}{llll}
\lambda & 0 & 1 & 0 \\
0 & \lambda & 0 & 1 \\
0 & 0 & \lambda & 0 \\
0 & 0 & 0 & \lambda
\end{array}\right) \text {, which has centraliser }\left(\begin{array}{llll}
a & b & ? & ? \\
c & d & ? & ? \\
0 & 0 & a & b \\
0 & 0 & c & d
\end{array}\right) \text {. }
$$

These centralisers can be seen to be big by taking a pair of 2 by 2 matrices generating a non abelian free group. In the first case we create a pair of 4 by 4 matrices doing the same by setting $a=1$ and placing each 2 by 2 matrix as the middle four entries, with all else set to zero. In the second case we put each 2 by 2 matrix both in the top left and bottom right hand places, with all other entries zero.

Note: Amongst the 4 by 4 blocks, we can distinguish between big and small centraliser according to whether the block does or does not satisfy the polynomial $(t-\lambda)^{2}$.

We can now prove our main result.

Theorem 5.4. Let $G$ be the amalgamated free product

$$
(F(X, Y) \times F(S)) *_{H_{1}=H_{2}}(F(U, V) \times F(T))
$$

where $F\left(X_{1}, \ldots, X_{n}\right)$ denotes the free group on elements $X_{1}, \ldots, X_{n}$ and $H_{1}=\left\langle A=X Y X^{-1} Y^{-1}, S\right\rangle \cong \mathbb{Z} \times \mathbb{Z} \cong H_{2}=\left\langle B=U V U^{-1} V^{-1}, T\right\rangle$ with the identification of $H_{1}$ to $H_{2}$ given by $B=A^{i} S^{j}$ and $T=A^{k} S^{l}$ for $i l-j k=1$ with $j, k$ odd integers and $i, l$ non zero even integers. Then $G$ is the fundamental group of a closed orientable graph 3-manifold but is not a subgroup of $G L(4, \mathbb{F})$ for $\mathbb{F}$ any field. 
Proof. By Corollary 4.2 we can assume that there is a faithful embedding of $G$ into $G L(4, \mathbb{F})$ where $\mathbb{F}$ has characteristic zero. Thus $G$ also embeds into $G L(4, \mathbb{C})$ and by a slight abuse of notation we denote the images of the group elements in $G L(4, \mathbb{F})$ by the same symbols. Then we can assume by Theorem 4.1 that all eigenvalues of $A, S, B, T$ are roots of unity. In particular none of these matrices is diagonalisable else it would be of finite order. However we can conjugate $G$ in $G L(4, \mathbb{C}$ ) such that $S$ is in Jordan normal form (although using our modified Jordan blocks rather than the standard ones where they differ). Thus $S$ is a direct sum of Jordan blocks as given above, but as the centraliser of $S$ contains a non abelian free group, we need to pick out at least one block from the lower list, which results in three cases. We start with the two cases where $S$ has only one eigenvalue.

Case 1:

$$
S=\left(\begin{array}{cccc}
\lambda & 0 & 0 & 1 \\
0 & \lambda & 0 & 0 \\
0 & 0 & \lambda & 0 \\
0 & 0 & 0 & \lambda
\end{array}\right) \text { and } F(X, Y) \text { is of the form }\left(\begin{array}{cccc}
a & ? & ? & ? \\
0 & ? & ? & ? \\
0 & ? & ? & ? \\
0 & 0 & 0 & a
\end{array}\right)
$$

which means that there is a homomorphism from $F(X, Y) \leq G L(4, \mathbb{C})$ to $G L(2, \mathbb{C})$ given by restriction to the middle 2 by 2 square of these matrices. As any element in the kernel would be upper triangular, this would result in a nilpotent kernel which contradicts Lemma 5.2 unless the homomorphism is injective. Moreover if we denote the image of $w \in F(X, Y)$ by $\bar{w}$, we have that $\bar{A}$ is a commutator $[\bar{X}, \bar{Y}]$ of 2 by 2 matrices $\bar{X}, \bar{Y}$ freely generating a free group. In particular $\operatorname{det}(\bar{A})=1$ but we do not have 1 as a repeated eigenvalue by Proposition 5.1 (i). However the eigenvalues $\rho, \rho^{-1}$ of $\bar{A}$ are also eigenvalues for $A$ so must be roots of unity by Theorem 4.1. But $\bar{A}$ has infinite order because the map from $F(X, Y)$ to $G L(2, \mathbb{C})$ sending $A$ to $\bar{A}$ is injective and a free group is torsion free. Thus we cannot have distinct eigenvalues, leaving only that $\bar{A}$ is conjugate to $\left(\begin{array}{rr}-1 & -b \\ 0 & -1\end{array}\right)$ for $b \neq 0$. Moreover the top and bottom diagonal entries of $A$ are 1 because $A=[X, Y]$ for $X, Y$ in the centraliser of $S$.

We now do some tidying up before proceeding to examine the forms of $B, T$ and the centraliser of $T$. 
Lemma 5.5. We can conjugate $G$ by a matrix in the centraliser of $S$ so that

$$
A=\left(\begin{array}{rrrr}
1 & 0 & ? & ? \\
0 & -1 & -b & ? \\
0 & 0 & -1 & 0 \\
0 & 0 & 0 & 1
\end{array}\right) \text { with } b \in \mathbb{C}-\{0\}
$$

Proof. First we conjugate using a 2 by 2 matrix in the middle block (and the identity outside this), so that $A$ is of the same form but with its middle block equal to $\bar{A}$. We then conjugate by elements of the form

$$
\left(\begin{array}{llll}
1 & \sigma & 0 & 0 \\
0 & 1 & 0 & 0 \\
0 & 0 & 1 & \tau \\
0 & 0 & 0 & 1
\end{array}\right) \text { to ensure that } A=\left(\begin{array}{llll}
1 & 0 & ? & ? \\
0 & ? & ? & ? \\
0 & ? & ? & 0 \\
0 & 0 & 0 & 1
\end{array}\right)
$$

which leaves the middle block of $A$ unaltered

Note that we have stayed within the centraliser of $S$, thus leaving it unchanged which means that

$$
B=A^{i} S^{j}=\left(\begin{array}{cccc}
\lambda^{j} & 0 & ? & ? \\
0 & \lambda^{j} & i b \lambda^{j} & ? \\
0 & 0 & \lambda^{j} & 0 \\
0 & 0 & 0 & \lambda^{j}
\end{array}\right) \text { and } T=A^{k} S^{l}=\left(\begin{array}{cccc}
\lambda^{l} & 0 & ? & ? \\
0 & -\lambda^{l} & -k b \lambda^{l} & ? \\
0 & 0 & -\lambda^{l} & 0 \\
0 & 0 & 0 & \lambda^{l}
\end{array}\right)
$$

where $\lambda$ is the eigenvalue of $S$. This can be shown using induction and recalling that $i$ is even and $k$ is odd. We now produce enough detail of the centraliser of $T$ to complete the argument in this case. (Actually the top right hand entry of $T$ must be zero, so that rank $\left(T-\lambda^{l} I\right)=2$ or else $T$ would not have a big centraliser but we will not use this fact.) If we work with 2 by 2 blocks in order to save excessive variable names, we are looking to see when

$$
\left(\begin{array}{c|c}
K & L \\
\hline M & N
\end{array}\right) \text { and } T=\left(\begin{array}{c|c}
J & E \\
\hline 0 & -J
\end{array}\right) \text { commute, where } J=\left(\begin{array}{rr}
\lambda^{l} & 0 \\
0 & -\lambda^{l}
\end{array}\right) \neq 0 .
$$

Now looking at the bottom left hand corner of the two equal products tells us that $M J=-J M$ so $M$ must have zeros on the diagonal. However from the top left of the products we have $K J-J K=E M$ and because the bottom 
left entry of $E$ is non zero, this forces the top right hand entry of $M$ to equal zero. Putting this back into $K J-J K=E M$ means that the top right hand entry of $K$ is also zero and the same argument provides the same conclusion for the top right hand entry of $N$. Thus elements commuting with $T$, and in particular $U$ and $V$, are all of the form

$$
\left(\begin{array}{llll}
? & 0 & ? & ? \\
? & ? & ? & ? \\
0 & 0 & ? & 0 \\
? & 0 & ? & ?
\end{array}\right)
$$

We note two points here: invariance of the second basis vector and that there is a homomorphism from the centraliser of $T$ to the entries in the four corners. Thus $B=[U, V]$ for $U, V$ of this form forces the entry in the second column of $B$ to be 1 , thus $\lambda^{j}=1$. But the kernel of our homomorphism is soluble, because commutators of commutators of elements in the kernel are all upper triangular. Thus by Lemma 5.2 the homomorphism is injective when restricted to $F(U, V)$ but the four corners of $B$ form the matrix $\left(\begin{array}{ll}1 & ? \\ 0 & 1\end{array}\right)$, which cannot be a commutator of a pair of elements in $G L(2, \mathbb{C})$ generating a non abelian free group by Proposition 5.1 (i).

Case 2:

$$
S=\left(\begin{array}{cccc}
\lambda & 0 & 1 & 0 \\
0 & \lambda & 0 & 1 \\
0 & 0 & \lambda & 0 \\
0 & 0 & 0 & \lambda
\end{array}\right) \text { and } F(X, Y) \text { is of the form }\left(\begin{array}{cccc}
a & b & ? & ? \\
c & d & ? & ? \\
0 & 0 & a & b \\
0 & 0 & c & d
\end{array}\right)
$$

Thus we have an obvious homomorphism from $F(X, Y)$ to $G L(2, \mathbb{C})$ by restriction to the top left top 2 by 2 block (which is equal to the bottom right block). Once again we denote the image of $w \in F(X, Y)$ by $\bar{w}$ and note that $A=[X, Y]$ implies that

$$
A=\left(\begin{array}{c|c}
{[\bar{X}, \bar{Y}]=\bar{A}} & ? \\
\hline 0 & {[\bar{X}, \bar{Y}]}
\end{array}\right)
$$

with $\bar{A}$ having determinant 1 . On conjugating $\bar{A}$ into Jordan normal form and building a block matrix in the centraliser of $S$ with this 2 by 2 conjugating element repeated twice on the diagonal and zeros elsewhere, we can assume 
that

$$
A=\left(\begin{array}{rrrr}
-1 & -b & a_{11} & a_{12} \\
0 & -1 & a_{21} & a_{22} \\
0 & 0 & -1 & -b \\
0 & 0 & 0 & -1
\end{array}\right) \text { and } T=\left(\begin{array}{cccc}
-\lambda^{l} & -k b \lambda^{l} & t_{11} & t_{12} \\
0 & -\lambda^{l} & t_{21} & t_{22} \\
0 & 0 & -\lambda^{l} & -k b \lambda^{l} \\
0 & 0 & 0 & -\lambda^{l}
\end{array}\right)
$$

as $k$ is odd. Now we know that $T$ must have a big centraliser. By the note immediately after Proposition 5.3, this forces $\left(T+\lambda^{l} I\right)^{2}=0$. This in turn implies that either $k b \lambda^{l}=0$ which is false or both $t_{21}=0$ and $t_{11}+t_{22}=0$ hold. At this point we felt it reasonable to revert to the computer. First suppose that $\bar{X}$ and $\bar{Y}$ are both in $S L(2, \mathbb{C})$. Using a similar block conjugating matrix as above, we can assume by Lemma 5.1 that

$$
X=\left(\begin{array}{cccc}
\frac{1+z^{2}}{w} & z & x_{11} & x_{12} \\
z & w & x_{21} & x_{22} \\
0 & 0 & \frac{1+z^{2}}{w} & z \\
0 & 0 & z & w
\end{array}\right) \text { and } Y=\left(\begin{array}{cccc}
\frac{1+w^{2}}{z} & -w & y_{11} & y_{12} \\
-w & z & y_{21} & y_{22} \\
0 & 0 & \frac{1+w^{2}}{z} & -w \\
0 & 0 & -w & z
\end{array}\right)
$$

On feeding this into Mathematica and asking for the (simplified version of) $A=X Y X^{-1} Y^{-1}$, we found that

$$
\begin{array}{r}
a_{11}+a_{22}=\frac{2\left(1+z^{2}+w^{2}\right)}{z w}\left(-x_{12} w-x_{21} w+2 y_{22} w+2 x_{22} z+y_{12} z+y_{21} z\right) \\
\text { and } a_{21}=-x_{12} w-x_{21} w+2 y_{22} w+2 x_{22} z+y_{12} z+y_{21} z
\end{array}
$$

so $a_{21}=0$ implies that $a_{11}+a_{22}=0$. Now it is easily shown by induction on $k$ and $l$ that $a_{21} \neq 0$ implies $t_{21} \neq 0$, in which case we have contradicted the condition above for $T$ to have a big centraliser. But again arguing by induction on $k$ and $l$, if $a_{21}=a_{11}+a_{22}=0$ then the same equations hold for the equivalent entries in all powers of $A$. Thus here $t_{11}+t_{22}=-2 l \lambda^{l-1}$ which is non zero as $l \neq 0$. Hence in all of these subcases the centraliser of $T$ is never big enough.

We did assume above that $\bar{X}, \bar{Y} \in S L(2, \mathbb{C})$ but for block matrices of the form $\left(\begin{array}{c|c}K & L \\ \hline 0 & K\end{array}\right)$ the determinant is $(\operatorname{det} K)^{2}$. Thus on being given $X, Y \in$ $G L(4, \mathbb{C})$ which both lie in the centraliser of $S$, we can multiply each by an appropriate scalar such that the repeated diagonal 2 by 2 block in each matrix has determinant 1 and the resulting commutator $X Y X^{-1} Y^{-1}$ will be unchanged. 
Case 3: The final case is where $S$ has more than one eigenvalue, hence we are putting Jordan blocks together. But we require at least one block from the lower list and at least one non diagonal block, which only allows for two eigenvalues $\lambda \neq \mu$ with

$$
S=\left(\begin{array}{cccc}
\lambda & 0 & 0 & 0 \\
0 & \lambda & 0 & 0 \\
0 & 0 & \mu & 1 \\
0 & 0 & 0 & \mu
\end{array}\right) \text { and } F(X, Y) \text { of the form }\left(\begin{array}{cccc}
? & ? & 0 & 0 \\
? & ? & 0 & 0 \\
0 & 0 & a & ? \\
0 & 0 & 0 & a
\end{array}\right)
$$

Again $\lambda, \mu$ are roots of unity and $A$ not of finite order and $\langle X, Y\rangle$ not soluble implies that we can conjugate to get

$$
A=\left(\begin{array}{cccc}
-1 & -b & 0 & 0 \\
0 & -1 & 0 & 0 \\
0 & 0 & 1 & 0 \\
0 & 0 & 0 & 1
\end{array}\right) \text { and } T=\left(\begin{array}{cccc}
-\lambda^{l} & ? & 0 & 0 \\
0 & -\lambda^{l} & 0 & 0 \\
0 & 0 & \mu^{l} & ? \\
0 & 0 & 0 & \mu^{l}
\end{array}\right)
$$

But both? are non zero so if $-\lambda^{l} \neq \mu^{l}$ then the centraliser of $T$ is too small. If however they are equal then $T$ can now be conjugated to have the same canonical form as $S$ did in Case 2. Therefore we may as well swap $S$ and $T$, which can be achieved by reversing the order of the two factors in the amalgamated product, thus keeping $G$ to be the same group but replacing the gluing matrix by its inverse. However the entries will still all be non zero and the same conditions on their parities will continue to hold.

\section{Further comments}

As mentioned in the introduction, it is only the linearity of fundamental groups of closed graph manifolds without a metric of non positive curvature which remains to be resolved. Now by [7] Example 4.1 the graph manifolds considered in this paper admit a metric of nonpositive curvature if and only if we have $\left(\begin{array}{ll}i & j \\ k & l\end{array}\right)=\left(\begin{array}{rr} \pm 1 & 0 \\ 0 & \pm 1\end{array}\right)$ or $\left(\begin{array}{rr}0 & \pm 1 \\ \pm 1 & 0\end{array}\right)$ (where we allow for all possible cases of signs) so the 3-manifolds in Theorem 5.4 do not possess metrics of non positive curvature and in particular linearity of their fundamental groups (over any field) is unknown. This suggests trying to increase 
the dimension above 4 in Theorem 5.4 and to this end we can automate the process in Section 3 to avoid ploughing through endless cases. We wrote a basic MAGMA program which on being given the two partitions of basis vectors for each eigenspace, outputted the determinant of the equations. For $i, j, k, l$ all non zero integers this was always non zero for dimension 5 . From this it is plausible that there is no embedding in $G L(n, \mathbb{C})$ for any $n$ where the peripheral elements are diagonalisable or have an eigenvalue which is not a root of unity. However given the variety of arguments that were employed in Section 5, we are much less sure whether these groups embed in $G L(5, \mathbb{C})$ or $G L(6, \mathbb{C})$ (say) if the peripheral elements are allowed to have more complicated Jordan normal forms.

We can also ask whether our 3-manifolds are fibred or virtually fibred over the circle. Now although we are in the class of closed graph manifolds without a metric of non positive curvature, these 3-manifolds can be fibred, such as a mapping torus of a Dehn twist on a closed surface, or not virtually fibred as shown in [9]. In fact our 3-manifolds are covered by [10] Theorem $\mathrm{D}$ and the example after Theorem $\mathrm{E}$ of the same paper, which combine to show that they are virtually fibred but not fibred.

We refer to [4] Section 9, especially Subsection 9.6 and Questions 9.7 and 9.14, for further information and problems on the linearity of 3-manifold groups.

\section{Appendix}

As claimed in Proposition 5.1, we need to show

Proposition 7.1. Given $A, B \in G L(2, \mathbb{C})$, let $C \in S L(2, \mathbb{C})$ be the commutator $A B A^{-1} B^{-1}$ and suppose that $C$ has repeated eigenvalues which must therefore be both 1 or both -1, thus on conjugating we can assume that $C= \pm\left(\begin{array}{ll}1 & k \\ 0 & 1\end{array}\right)$ for $k \in \mathbb{C}$

(i) If $C=\left(\begin{array}{ll}1 & k \\ 0 & 1\end{array}\right)$ for $k \neq 0$ then both $A$ and $B$ are already upper triangular too, thus $\langle A, B\rangle$ is a soluble group.

(ii) If $A, B$ are in $S L(2, \mathbb{C})$ and of infinite order with $C=\left(\begin{array}{rr}-1 & -k \\ 0 & -1\end{array}\right)$ for some $k \neq 0$ then we can simultaneously conjugate $A$ and $B$ in $S L(2, \mathbb{C})$ such 
that $A B A^{-1} B^{-1}$ is as above (though for a possibly different $k \in \mathbb{C}$ ) and

$$
A^{-1} B^{-1} A B=\left(\begin{array}{rr}
-1 & 0 \\
k & -1
\end{array}\right)
$$

whereupon there exist $z, w \in \mathbb{C}-\{0\}$ such that

$$
A=\left(\begin{array}{cc}
\frac{1+z^{2}}{w} & z \\
z & w
\end{array}\right), \quad B=\left(\begin{array}{cc}
\frac{1+w^{2}}{z} & -w \\
-w & z
\end{array}\right) \text { with } k=\frac{2\left(1+z^{2}+w^{2}\right)}{z w} .
$$

Proof. For (i), set $A=\left(\begin{array}{ll}a & b \\ c & d\end{array}\right)$ and $B=\left(\begin{array}{ll}e & f \\ g & h\end{array}\right)$. Then the matrix $(C-I) B A=A B-B A$ has zeros on the bottom row which implies that $c f=b g$ and $c(e-h)=g(a-d)$. Multiplying the second equation by $f$ tells us that $g(b(e-h)-f(a-d))=0$. If the latter factor is zero then the top right hand entry of $A B-B A$ is zero but this in turn forces $A B=B A$. Thus $g=0$ but if $c \neq 0$ then both $f$ and $e-h$ are zero, which would make $B=h I$ and hence $C=I$.

For (ii) we first conjugate $A^{-1} B^{-1} A B$, which is itself a conjugate of $C$ thus has -1 as a repeated eigenvalue, by a matrix of the form $\left(\begin{array}{ll}1 & x \\ 0 & 1\end{array}\right)$ so that $A^{-1} B^{-1} A B=\left(\begin{array}{rr}-1 & 0 \\ -l & -1\end{array}\right)$ and $C$ is unchanged. Then we can further conjugate by $\left(\begin{array}{ll}t & 0 \\ 0 & t^{-1}\end{array}\right)$ to make $k=l$. This can be done unless the bottom left hand entry of $A^{-1} B^{-1} A B$ is also zero (in which case $A B A^{-1} B^{-1}=-I$ ).

We now proceed in two stages. First we set $X=A B, Y=A^{-1} B^{-1}$ and prove the following lemma.

Lemma 7.2. If $X, Y \in S L(2, \mathbb{C})$ with

$$
Y X=\left(\begin{array}{rr}
-1 & 0 \\
-k & -1
\end{array}\right) \quad, \quad X Y=\left(\begin{array}{rr}
-1 & k \\
0 & -1
\end{array}\right) \text { for } k \neq 0
$$

then $X$ and $Y$ (or $-X$ and $-Y$ ) are of the form

$$
X=\left(\begin{array}{rr}
e & -1 \\
1 & 0
\end{array}\right) \quad, \quad Y=\left(\begin{array}{rr}
0 & -1 \\
1 & d
\end{array}\right)
$$

for $d, e \in \mathbb{C} \backslash\{0\}$. 
Proof. We now set

$$
Y=\left(\begin{array}{ll}
a & b \\
c & d
\end{array}\right) \quad, \quad X=\left(\begin{array}{ll}
e & f \\
g & h
\end{array}\right)
$$

Then the zero coefficients give

$$
\begin{aligned}
& a f+b h=0 \\
& a g+c h=0 .
\end{aligned}
$$

First assume $h \neq 0$. Then we have $f \neq 0$ (since $f=0$ implies that $b=0$, otherwise $X$ is a singular matrix, but then looking at $X Y$ tells us that $k=0)$.

So writing $a / h=-b / f=-c / g=\lambda$ gives

$$
Y=\left(\begin{array}{cc}
\lambda h & -\lambda f \\
-\lambda g & d
\end{array}\right)
$$

and equating the two -1 coefficients in $Y X$ gives $\lambda h e=d h$, so $d=\lambda e$. But then $Y= \pm X^{-1}$, contradicting $k \neq 0$.

Thus we can only have $h=0$, and $a=0$, giving $c=-1 / b$ and $g=-1 / f$. Again, equating the -1 coefficients in $Y X$ gives $b=f$, and comparing the $-k$ and $k$ coefficients in $Y X$ and $X Y$ respectively tells us that

$$
b(d+e)=\frac{d+e}{b} .
$$

If $d=-e$ then $k$ would be 0 , so we can take $b=-1$ and the lemma is proved.

Now we repeat the process, setting

$$
A=\left(\begin{array}{ll}
\alpha & \beta \\
\gamma & \delta
\end{array}\right) \quad, \quad B=\left(\begin{array}{ll}
\epsilon & \zeta \\
\eta & \theta
\end{array}\right)
$$

with

$$
A B=X=\left(\begin{array}{rr}
e & 1 \\
-1 & 0
\end{array}\right) \quad, \quad B A=Y^{-1}=\left(\begin{array}{rr}
d & -1 \\
1 & 0
\end{array}\right) .
$$

Again the zero coefficients tell us that

$$
\begin{aligned}
& \gamma \zeta+\delta \theta=0 \\
& \eta \beta+\delta \theta=0
\end{aligned}
$$


so $\gamma \zeta=\eta \beta$.

If say $\beta=0$, then taking $\theta=0$ and $\gamma=0$ gives us a "singular solution" where $A$ has order 4 and we argue the same way for any other combination of zeros.

Otherwise we write $\beta=\mu \gamma, \zeta=\mu \eta$, and then comparing the 1 coefficients in $X$ and $Y^{-1}$ tells us

$$
\mu(\alpha \eta+\gamma \theta)=1=\alpha \eta+\gamma \theta
$$

and $\mu=1$. We can now set $z=\zeta=\eta$ and $w=-\beta=-\gamma$. Then by using the fact that $\operatorname{det}(A)=\operatorname{det}(B)=1$, we are left with the four equations

$$
\begin{aligned}
\delta \theta & =w z \\
\alpha \delta-w^{2} & =1 \\
\epsilon \theta-z^{2} & =1 \\
z(\alpha+\delta) & =w(\epsilon+\theta)
\end{aligned}
$$

and we then eliminate first $\theta$, then $\alpha$ and finally $\epsilon$ to obtain $\delta^{2}=z^{2}$. We take $\delta=z$, so $\theta=w$ and $\alpha=\left(1+w^{2}\right) / z, \epsilon=\left(1+z^{2}\right) / w$.

In the above proposition, part (i) is a folklore result although we could not locate a short proof in the literature. Part (ii) appears as the appendix in the author's $\mathrm{PhD}([5])$ but as this does not appear to be generally available online, we include it here too.

\section{References}

[1] I. Agol, Criteria for virtual fibering, J. Topol. 1 (2008), 269-284.

[2] I. Agol, The virtual Haken conjecture, (2012) http://arxiv.org/abs/1204.2810

[3] M. Aschenbrenner and S. Friedl, 3-Manifold Groups are Virtually Residually p, Memoirs Amer. Math. Soc., Vol 225, No 1058 (2013)

[4] M. Aschenbrenner, S. Friedl and H. Wilton, 3-manifold groups, (2013) http://arxiv.org/abs/1205.0202v3 
[5] J.O. Button, Quasi-Fuchsian space of the once-punctured Torus, PhD, University of Warwick (1994)

[6] J. Hempel, Residual finiteness for 3-manifolds, Combinatorial group theory and topology (Alta, Utah, 1984), 379-396, Ann. of Math. Stud. 111, Princeton Univ. Press, Princeton, NJ, 1987.

[7] B. Leeb, 3-manifolds with(out) metrics of non positive curvature, Invent. Math. 122 (1995), 277-289.

[8] Yi Liu, Virtual cubulation of nonpositively curved graph manifolds, J. Topol., to appear (2013)

[9] J. Luecke and Ying-Qing Wu, Relative Euler number and finite covers of graph manifolds, Geometric topology (Athens, GA, 1993), 80-103, AMS/IP Stud. Adv. Math. 2.1, Amer. Math. Soc., Providence, RI, 1997.

[10] W. D. Neumann, Commensurability and virtual fibration for graph manifolds, Topology 36 (1997), 355-378.

[11] P. Przytycki and D.T.Wise, Mixed 3-manifolds are virtually special, (2012)

http://arxiv.org/abs/1205.6742

[12] D. T. Wise, From Riches to Raags: 3-Manifolds, Right-Angled Artin Groups, and Cubical Geometry, CBMS Regional Conference Series in Mathematics No. 117, American Mathematical Society, Providence, RI, 2012. 